\title{
A friction test between steel and a brittle material at high contact pressures and high sliding velocities
}

\author{
B. Durand ${ }^{1,2}$, P. Bailly ${ }^{1}$, F. Delvare ${ }^{1}$, and D. Picart ${ }^{2}$ \\ ${ }^{1}$ ENSI Bourges, Laboratoire PRISME, 18020 Bourges, France \\ 2 CEA, DAM, Le Ripault, 37260 Monts, France
}

\begin{abstract}
Our aim is to characterize the interface behaviour between an aggregate material and steel. This work focuses on contact pressures and sliding velocities reaching $100 \mathrm{MPa}$ and $10 \mathrm{~m} / \mathrm{s}$. The set-up consists in a cylindrical sample of the aggregate material which slips into a steel tube. The tube is both a confinement vessel and a sliding surface. Thanks to confinement, the material can be tested under high stresses without failure. The interface pressure is generated by an axial compression. The sample is pressed on a spring, so it can be simultaneously compressed and rubbed on the tube. The set-up has been tested in the case of a quasi-static loading and the $100 \mathrm{MPa}$ pressure has been reached. Then the set-up was mounted on a Split Hopkinson Pressure Bar device in order to reach higher velocities. Numerical simulations have been realized to check the feasibility and the relevance of this dynamic test. These results are analysed and compared to the experimental ones.
\end{abstract}

\section{Introduction}

The ignition of a plastic bonded explosive submitted to an impact depends strongly on the contact conditions at the boundaries. As a result, a perfect knowledge of the interface behaviour is necessary to predict exactly the evolution of such a set [1-4]. The aim, to take up this challenge, is to design a device to study the friction between steel and an explosive under the conditions of an impact: contacts pressures and sliding velocities reaching respectively $100 \mathrm{MPa}$ and $10 \mathrm{~m} / \mathrm{s}$.

A specific apparatus is required to simultaneously obtain high pressures and high velocities. Several techniques have been developed to measure friction coefficients under these conditions: explosively driven friction test [5], plate impact [6], torsional Kolsky bar set-up [6-9], dynamometer ring with a rectangular specimen launched by a gas gun or a hydraulic machine [10] and pin-on-disk apparatus [3].

For safety reasons, the presented experiments have been carried out with an inert material, denoted I1, whose mechanical properties are close to explosives ones. It is a fine powder mixed with a binder which has been compacted. Its mechanical behaviour has been previously studied [11]. Its elastic properties are a 0.4 Poisson's ratio and a $3 \mathrm{GPa}$ Young's modulus. It is a quasi-brittle material with a Drucker-Prager's threshold. Let $\underline{\sigma}, P$ and $Q$ respectively denoting the stress tensor, the hydrostatic pressure and the octahedral scission:

$$
\begin{gathered}
P=\frac{1}{3} \operatorname{tr}(\underline{\underline{\sigma}}) \\
Q=\sqrt{\frac{1}{3}(\underline{\underline{\sigma}}-P \underline{I}):(\underline{\underline{\sigma}}-P \underline{I})}
\end{gathered}
$$

where $\underline{I}$ is the identity tensor

The behaviour remains elastic if the following criterion is respected:

$$
Q+B P<A
$$

The cohesive stress $A$ depends on the strain rate. It varies from $12 \mathrm{MPa}$ at low strain rates to $40 \mathrm{MPa}$ at the strain rates reached during Split Hopkinson Pressure Bar (SHPB) tests, typically $100 \mathrm{~s}^{-1}$. The internal friction coefficient $B$ is equal to 0.3 . In the case of a uniaxial compressive loading, the criterion corresponds to a maximum axial stress of $30 \mathrm{MPa}$ at low strain rates and $100 \mathrm{MPa}$ at the strain rates reached during SHPB tests. As the material has a brittle behaviour, contact pressures higher than the compressive strength can lead to an unexpected fracture and thus to erroneous measurements.

Quasi-oedometric tests consist in confining a cylindrical sample in a rigid ring [11-13] and applying high stresses on brittle materials without reaching their shear resistance criterions. Indeed, under increasing magnitudes of hydrostatic pressures, materials with a Drucker-Prager's threshold can reach higher octahedral scissions.

The proposed set-up is thus based on a quasi-oedometric configuration. A SHPB device is used to obtain the high velocities.

\section{Set-up}

A previous study of the influence of the sliding velocity on the friction has been performed [14]. The axial compression of the sample was applied by a clamping screw but the interface pressure remained too low (the screw breaks). A new system has been designed in order to reach higher interface pressure (figure 1): the specimen is now pressed on a spring, so it can be simultaneously compressed and rubbed on the tube. The spring is a washers assembly. Its flexibility allows a sufficient displacement to ensure the same friction conditions on the entire interface. It must be highlighted that unlike classical tribometers, such a system allows to obtain the normal pressure and the friction with the same actuator.

The two longitudinal strain gauges glued on the aluminium rod are diametrically opposed to eliminate the flexural strains caused by dissymmetry. The aggregate 


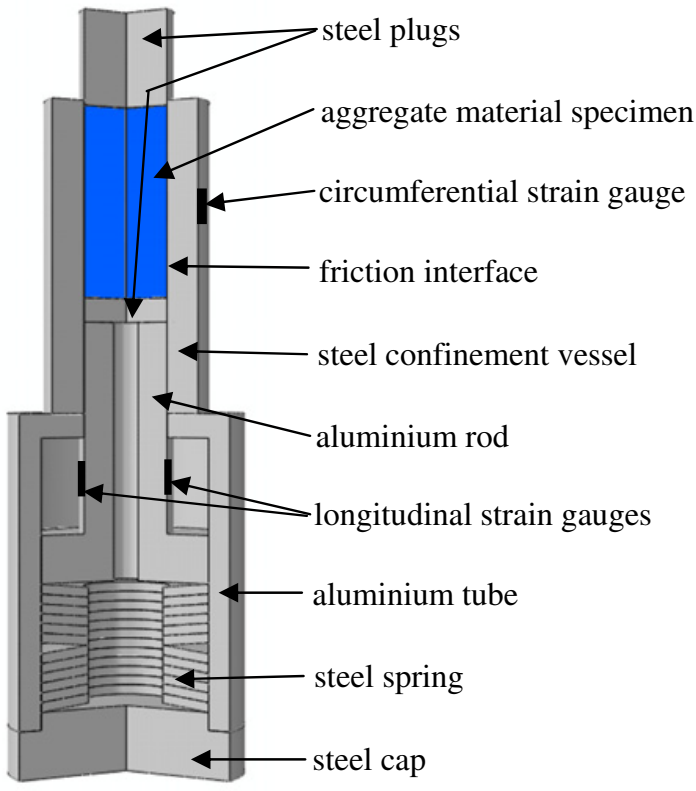

Fig. 1. Sketch of the confined friction set-up.

material specimen has a diameter of $20 \mathrm{~mm}$ and is $40 \mathrm{~mm}$ long. The steel confinement vessel is made of steel (the Young's modulus is $210 \mathrm{GPa}$ and the Poisson's ratio is 0.33 ). Its outer diameter is $35 \mathrm{~mm}$.

\section{Experimental results and numerical analysis}

The confined friction set-up is mounted on a testing machine and on a SHPB system. Friction tests are conducted at different sliding velocities.

Both tests are modelled with the finite element method to identify friction parameters. The simulations were performed using ABAQUS CAE with an axisymmetry assumption.

\subsection{Quasi-static test}

Thanks to the spring flexibility, the sliding velocities reached at the friction interface are of the order of the velocity imposed by the machine to the assembly, i.e. $1 \mathrm{~mm} / \mathrm{min}$.

The testing machine sensor indicates the force applied by the machine $F_{m}$. Moreover, the strains measurements on the aluminium rod provide the value of the normal force in this component, denoted $F_{r o}$. Experimental measurements are displayed on figure 2 .

Figure 2 shows $F_{r o}$ as a linear function of $F_{m}$. The value of ratio is linked to the interface behaviour.

Numerical simulations are performed using an implicit code. A Coulomb's friction law is imposed at the interface between the specimen and the tube: the ratio between the tangential stress and the normal pressure is assumed equal to a constant coefficient $f$ during a friction process. The simulations also show a linear dependence of the force applied by the machine on the normal force in the rod. The

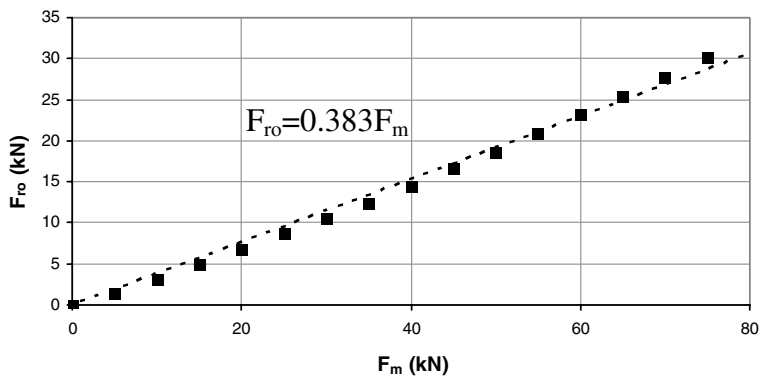

Fig. 2. Experimental evolutions of $F_{m}$ and $F_{r o}$ respectively the force applied by the machine and the normal force in the rod.

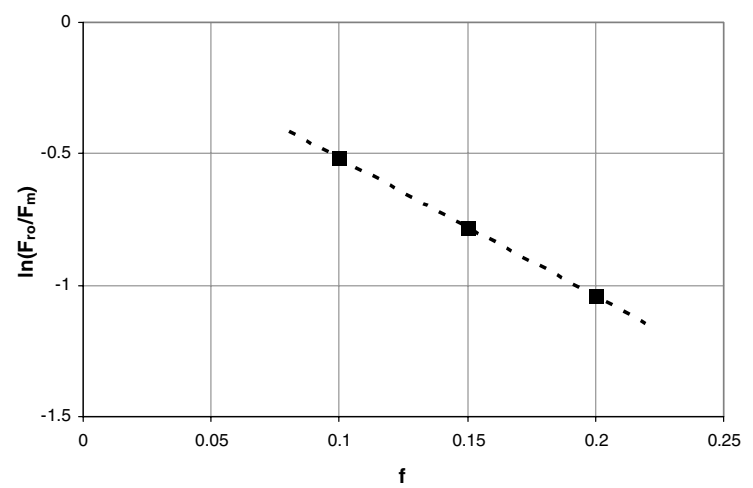

Fig. 3. Numerical relation between the logarithm of the forces ratio $\ln \left(F_{r o} / F_{m}\right)$ and the friction coefficient $f$.

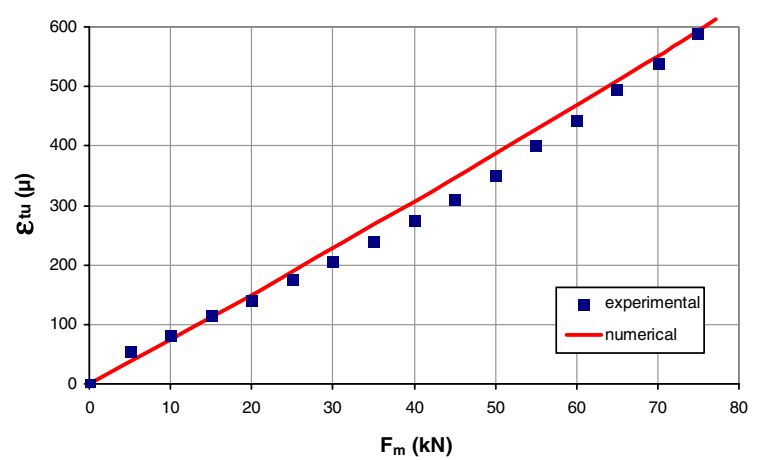

Fig. 4. Comparison between the experimental and the numerical strain on the tube.

numerical relation between the forces ratio and the friction coefficient $f$ is determined and presented on figure 3 .

This numerical relation enables to determine the friction coefficient from the experimental value of the force ratio. $f=0.18$ is obtained with $F_{r o} / F_{m}=0.383$ as on figure 2.

Numerical simulations are run with this value of the friction coefficient to check the model validity. The external circumferential strains of the tube $\varepsilon_{t u}$ are calculated and these numerical results are compared to the corresponding measurements.

Figure 4 shows that the experimental and the numerical values of the strain $\varepsilon_{t u}$ are in good agreement.

Finite element calculations have been performed using an elacticity assumption. As the aggregate material has a low strength, failure can occur and can lead to an erreneous 


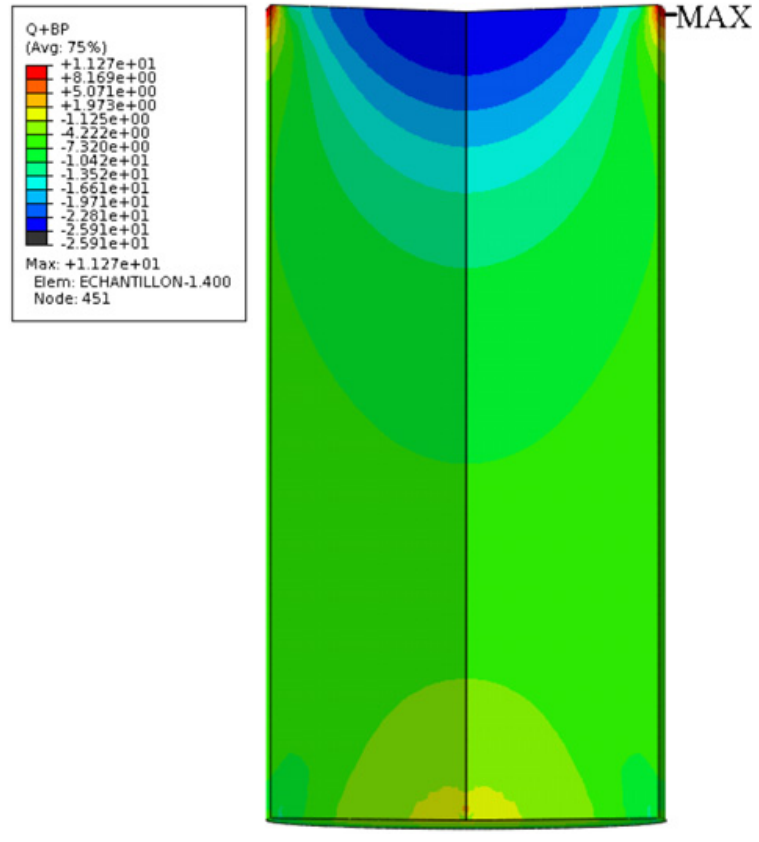

Fig. 5. Sketch of the sample with the values of $Q+B P$ at the maximal load. The maximal value is approximatively $11 \mathrm{MPa}$.

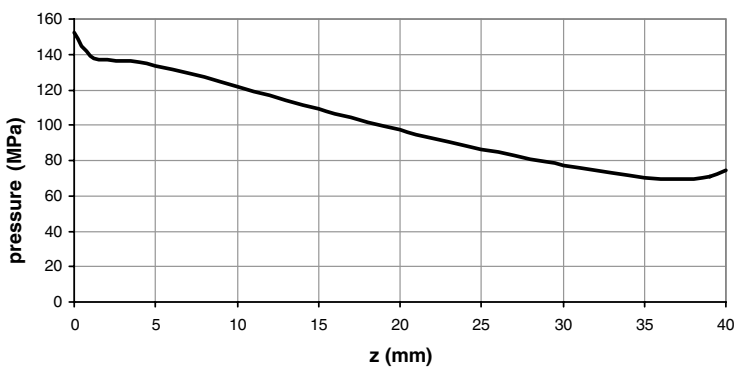

Fig. 6. Contact pressure along the interface at the maximum load. The position $z=0$ corresponds to the top of the specimen (figure 1).

calculation. $Q+B P$ must therefore be calculated in the sample to verify the assumption.

According to finite element simulations, the criterion (3) is respected everywhere in the specimen. It confirms the elasticity assumption consistency.

The contact pressure is numerically determined at the interface. A strongly heterogeneous distribution is obtained. Figure 6 shows that pressure has reached the desired order of magnitude. The high value of the Poisson's ratio is an advantage.

\subsection{Dynamic test}

The static test analysis showed that high pressure can be generated with this configuration. Our aim is now to combine high pressure and high velocity. The friction setup is then mounted on a SHPB device. Bars are made of aluminium (Young's modulus equal to $74 \mathrm{GPa}$ and Poisson's ratio equal to 0.33) and have a diameter of $40 \mathrm{~mm}$. The striker, the input bar and the output bar have

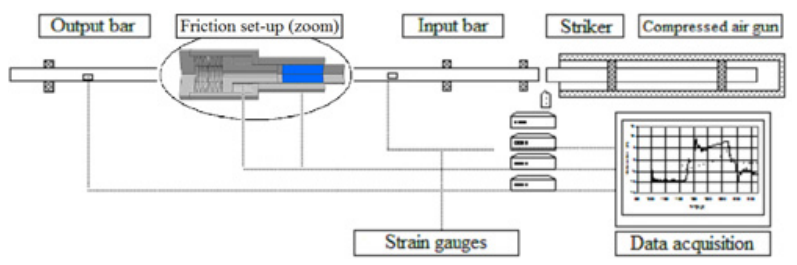

Fig. 7. Schematic of the friction set-up mounted between the bars.

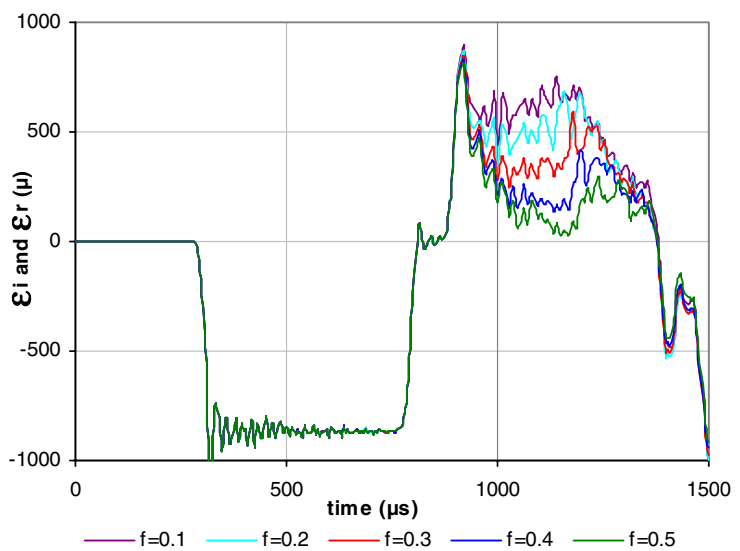

Fig. 8. Numerical evolution of the strain at the middle of the input bar. The first part corresponds to the incident wave and the second ones to the reflected waves, depending of $f$ magnitude.

respectively a length of $1.25 \mathrm{~m}, 3 \mathrm{~m}$ and $1.8 \mathrm{~m}$. The way the new apparatus is assembled is detailed on figure 7.

The impact of the striker results in an incident compressive wave $\varepsilon_{i}$ in the input bar. Wave reverberations occur in the set-up and it results in a transmitted wave $\varepsilon_{t}$ in the output bar and in a reflected wave $\varepsilon_{r}$ in the input bar. The incident and the reflected longitudinal strain waves have to be measured at a place where they are separated in the time. The gauge is therefore glued at the middle of the input bar. The circumferential strain on the tube $\varepsilon_{t u}$ is also recorded (figure 1).

The incident strain wave depends only on the impact velocity and on the characterics of the striker and of the input bar. It does not depend on phenomena occurring in the friction set-up. The other strains depend on the set-up behaviour: they can be seen as the mechanical response of the friction set-up to the imposed incident strain.

Finite element simulations of the experiment have been performed using ABAQUS. Several friction coefficients $f$ have been used to study the influence of the friction on the mechanical response.

Figures 8-10 represent the numerical strains which should be experimentally measured. Several friction conditions have been used with the same incident wave. The value of the friction coefficient has a noticeable influence on the mechanical response of the set-up.

The dynamic friction coefficient is identified by fitting as well as possible the numerical and the experimental results. The following figures give some comparisons between the experimental strains and the numerical ones obtained with $f=0.4$ which seems to be the best fit. Additional simulations of the experiment with no contact 


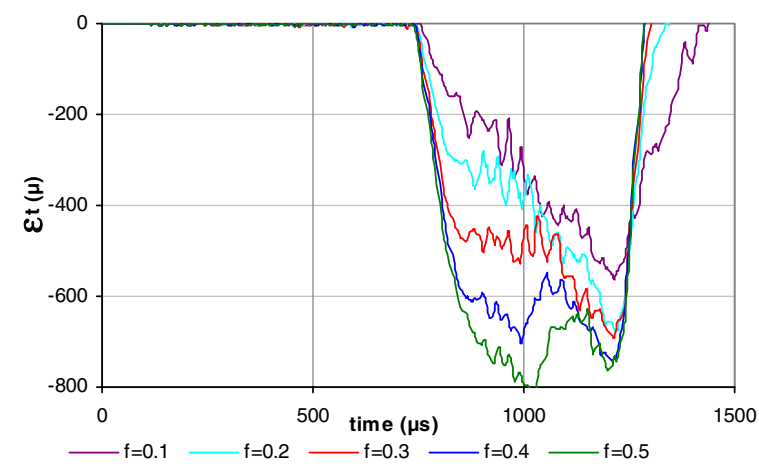

Fig. 9. Numerical evolution of the strain of the output bar at the position of the gauge (500 $\mathrm{mm}$ from the set-up). The waves correspond to the transmitted waves, depending on $f$ magnitude.

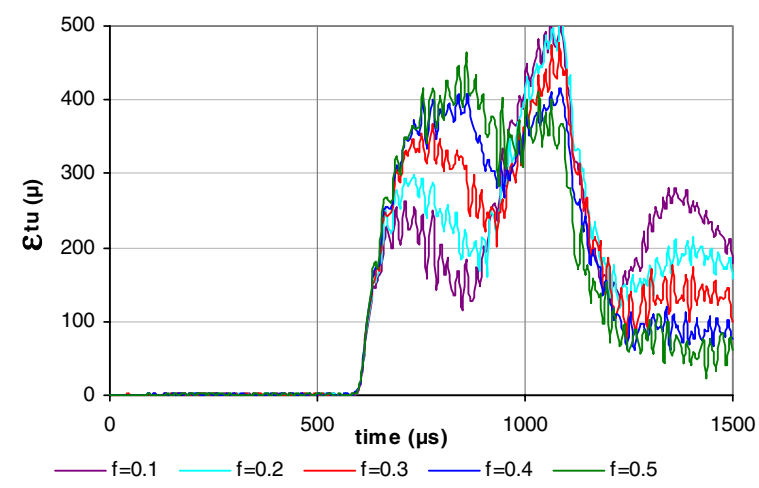

Fig. 10. Numerical evolution of the circumferential strain on the tube (at the position of the gauge), depending on $f$ magnitude.

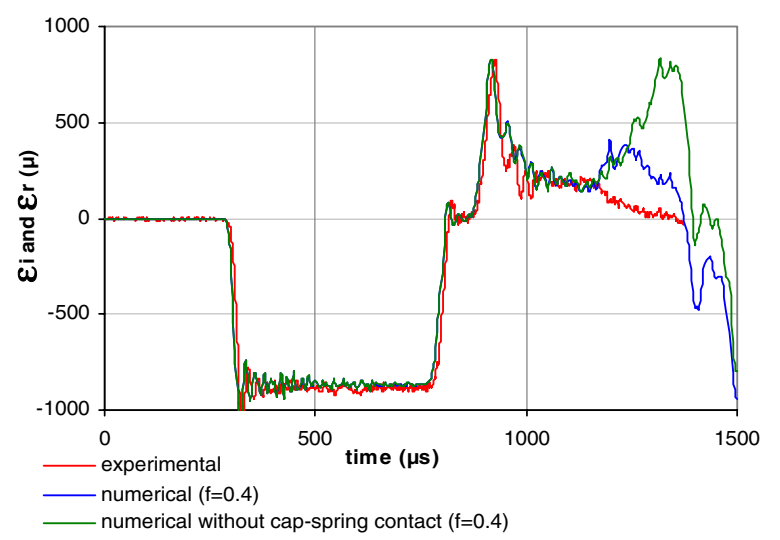

Fig. 11. Numerical and experimental evolutions of the strain at the middle of the input bar.

between the spring and the cap (figure 1) were also performed to understand the influence of the spring.

Comparisons between the numerical results obtained with and without the spring-cap contact on figures 11-13 show that spring stretching has not an immediate influence on the mechanical response. At the beginning of the test, the compression of the sample is actually due to the inertia of the rod and the spring.

The numerical results obtained with $f=0.4$ are in good agreement with the experimental results but only at the beginning of the signals. The analysis of the tube strain and the reflected strain displays that the experimental

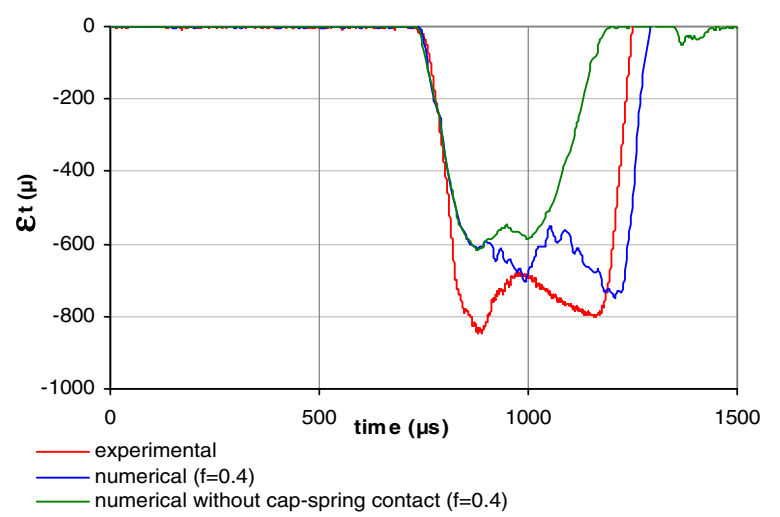

Fig. 12. Numerical and experimental evolutions of the strain of the output bar (at the position of the gauge).

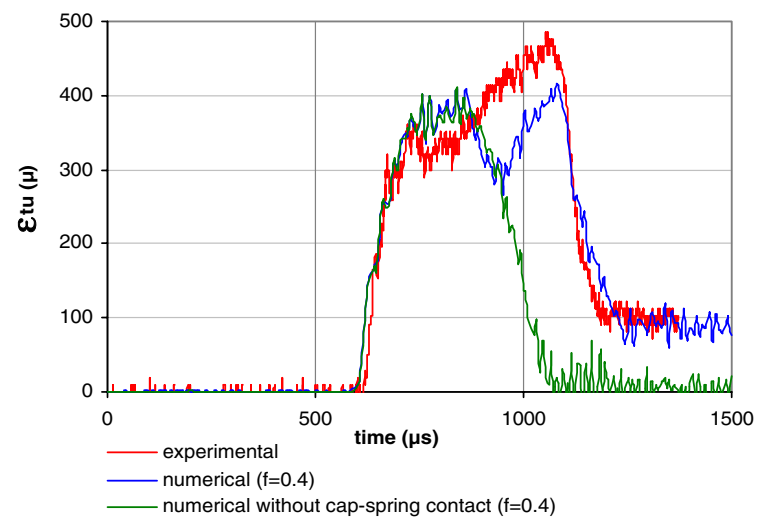

Fig. 13. Numerical and experimental evolutions of the circumferential strain on the tube (at the position of the gauge).

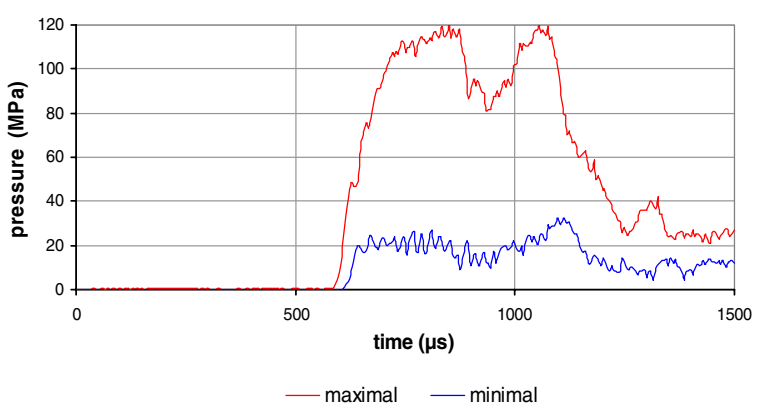

Fig. 14. Numerical evolution of the pressure (extreme values along the interface).

and the numerical results begin to differ slightly before the appearance of the spring-cap contact influence. It is very noticeable in the case of the reflected wave. The discrepancy between experiment and simulation could therefore be explained by a non adapted modelling of the spring stretching.

However, the beginnings of the experimental signals prove that the dynamic friction coefficient is higher than the static one. Indeed, the numerical simulation with $f=$ 0.2 close to the static coefficient $f=0.18$ gives different results from those obtained with $f=0.4$ which are the closest to dynamic experiment.

The interface pressure is between 20 and $120 \mathrm{MPa}$ (figure 14). According to the simulations, sliding velocities are heterogeneous at the very beginning to the test. They 


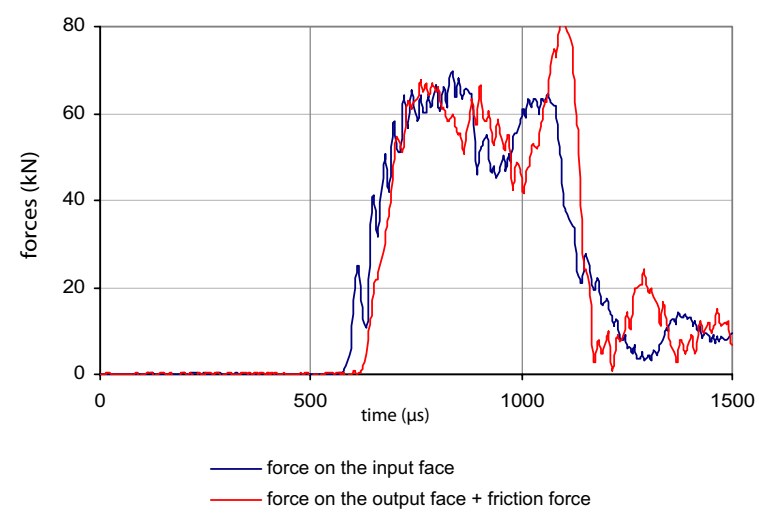

Fig. 15. Numerical evolution of the forces applied on the specimen. "Input face" denotes the specimen face on the input bar side and "output face" denotes the specimen face on the output bar side.

reach an approximate value of $2-3 \mathrm{~m} / \mathrm{s}$ before the appearance of the spring-cap contact influence.

A satisfactory equilibrium of the specimen is shown on figure 15 .

\section{Conclusion}

In quasi-static loading conditions, the tribometer enables a reliable identification of the interface behaviour between the aggregate material and steel. High interface pressures can be easily generated during the friction process thanks to the original configuration.

An increase of the friction with the sliding velocity is established. It is coherent with [14]. The experiments performed during our first study and those presented in this paper show how it is difficult to apply simultaneously high pressures and high velocities. Indeed, in [14], velocities were of the order of $10 \mathrm{~m} / \mathrm{s}$ but mean pressures did not exceed $20 \mathrm{MPa}$. On the contrary, in this paper, pressures reach $100 \mathrm{MPa}$ but velocities drop to $2-3 \mathrm{~m} / \mathrm{s}$.

Simulations and experiments analyses show that the tribometer presented here could be improved in dynamics. Firstly, the use of a spring to simultaneously compress and rub the sample on the tube is not so relevant. Indeed, as wave propagation is not immediate, the influence of the spring stretching is strongly delayed. Moreover, phenomena linked to wave propagation make the behaviour of the spring complex during the stretching phase.

\section{References}

1. D. Picart, F. Delmaire-Sizes, C. Gruau, H. Trumel, Ignition of a HMX-based PBX submitted to impact: strain localisation and boundary condition, $16^{\text {th }}$
Conference of the American Physical Society Topical Group on Shock Compression of Condensed Matter, (2009)

2. Hoffman, J.B. Chandler, Aspect of the tribology of the plastic bonded explosive LX-04, Propellants, Explosives, Pyrotechnics, 29, 368-373, (2004)

3. P.M. Dickson, G.R. Parker, L.B. Smilowitz, J.M. Zucker, B.W Asay, Frictional Heating and Ignition of Energetic Materials, CP845, Conference of the American Physical Society Topical Group on Shock Compression of Condensed Matter, 1057-1060, (2005)

4. C. Gruau, D. Picart, R. Belmas, E. Bouton, F. Delmaire-Sizes, J. Sabatier, H. Trumel, Ignition of a confined high explosive under low velocity impact, International Journal of Impact Engineering, 36, 537-550, (2008)

5. H.J. Kim, A. Emge, R.E. Winter, P.T. Keightley, W.K. Kim, M.L Falk, D.A. Rigney, Nanostructures generated by explosively driven friction: Experiments and molecular dynamics simulations, Acta Materiala, 57, 5270-5282, (2009)

6. S. Rajagopalan, M.A. Irfan, V. Prakash, Novel experimental techniques for investigating time resolved high speed friction, Wear, 225-229, 1222-1237, (1999)

7. S. Rajagopalan, V. Prakash, An experimental method to study high speed sliding characteristics during forward and reverse slip, Wear, 249, 687-701, (2001)

8. H. Huang, R. Feng, Dynamic tribological response of SiC fracture surfaces, Mechanics of Materials, 38, 186-202, (2006)

9. H. Huang, R. Feng, Dynamic Friction of SiC Surfaces: A Torsional Kolsky Bar Tribometer Study, Tribology Letters, 27, 329-338, (2007)

10. S. Philippon, G. Sutter, A. Molinari, An experimental study of friction at high sliding velocities, Wear, 257, 777-787, (2004)

11. P. Bailly, F. Delvare, J. Vial, J.L. Hanus, M. Biessy, D. Picart, Dynamic behavior of an aggregate material at simultaneous high pressure and strain rate: $S H P B$ triaxial tests, International Journal of Impact Engineering, 38, 73-84, (2011)

12. P. Forquin, A. Arias, R. Zaera, An experimental method of measuring the confined compression strenght of geomaterials, International Journal of Solids and Structures, 44, 4291-4317, (2007)

13. P. Forquin, K. Safa, G. Gary, Influence offree water on the quasi-static and dynamic of strength of concrete in confined compression tests, Cement and Concrete Research, 40, 321-333, (2009)

14. B. Durand, F. Delvare, P. Bailly, D. Picart, Caractérisation du comportement à l'interface entre un matériau agrégataire et un acier sous forte pression et à grande vitesse, $20^{\text {iéme }}$ Congrès Français de Mécanique, (2011), in french. 